\title{
Design of transfer function detecting device for large aperture optical
}

\author{
system \\ Yufeng Yan ${ }^{1, a^{*}}$, Zhenzhen Bian ${ }^{1, b}$, Liyan Yang ${ }^{1, c}$, Xiang Zhou ${ }^{1, d}$ \\ ${ }^{1}$ Changchun University of Science and Technology, Changchun130022, China \\ a22477675@qq.com, bbzz_cust@126.com, ${ }^{c} 1051531588 @ q q . c o m,{ }^{d} x z$ adorable@qq.com
}

Keyword: Transfer function; Off-axis reflection; Large caliber; Detection

Abstract. The transfer function is the performance of an optical System's Imaging quality. It's an important indicator of an optical system. Detecting the transfer function plays a particularly important role in the project processing and finishing process of the optical system. In order to detect the large caliber optical system's transfer function, this paper designs a detection systems, who's focal length is $2000 \mathrm{~mm}$ and caliber is $500 \mathrm{~mm}$, for detecting the transfer function of different optical systems who has large Caliber. This system's light source can be replaced for measuring the different optical systems .It suits for a lot of optical systems, who's caliber is from 1 500mm. It depends on the Off-axis reflective optical system. It has a lot of advantages, e.g. it does not make any chromatic aberration, it can be lightweight and has no center block.

\section{Introduction}

In recent years, the application of complex and large aperture optical system is becoming more and more wide, and the imaging quality of optical system is becoming more and more important. So it is very important to detect the transfer function of an optical system. Traditional optical detection technology is facing great challenges in the detection of large aperture optical system's transfer function. In this case, it is necessary to research the transfer function detection technology of large aperture optical system.

This article designs a large reflection optical collimator and the light source's optical system to meet the needs of testing the large aperture optical system transfer function. The device makes a specific diameter of parallel light through the system under test. The CCD receives the image, and the image and the image spectrum accepted will be analyzed by the computer. Then the transfer function of the large aperture optical system is obtained by comparing the calculation.

\section{detection principle}

In this device, the transfer function of the optical system is detected by the method of Pinhole image features. The "pinhole" by the light source and the collimation system is imaged by optical system to be measured. Images collected by CCD. The energy distribution of the system is directly related to the characteristics of the system, and the imaging quality of the system is measured.

The characteristic spectrum $F(u, v)$ is obtained by light distribution of the "pinhole" $f(x, y)$ by Fourier transformation.CCD collects the optical energy distribution of the target image. Through computer processing, frequency spectrumG(u, v) can be obtained by Fourier transformation. According to the linear invariant system theory, the transfer function $\operatorname{OTF}(\mathrm{u}, \mathrm{v})$ of optical system to be measured is obtained. 


$$
\operatorname{OTF}(u, v)=\frac{G(u, v)}{F(u, v)}
$$

If $\mathrm{x}, \mathrm{y}$ is a reference image coordinate, and $\mathrm{u}, \mathrm{v}$ are the spatial frequency of $\mathrm{x}, \mathrm{y}$ direction.

The light distribution of the "pinhole" can be represented by a two dimensional delta function.

$$
f(x, y)=\delta(x, y)
$$

After being imaged by the system, the image of the image is distributed as the impulse response of the optical system, which is the point spread function (PSF):

$$
g(x, y)=h(x, y)=\operatorname{PSF}(x, y)
$$

The point spread function is obtained by the two-dimensional Fourier Transformation:

$$
\xi(\operatorname{PSF} x, y))=M T H \zeta, \eta) \mathrm{e}^{\mathrm{iPTF}(\zeta, \eta)}
$$

The image detection method can be used to measure the two-dimensional optical transfer function of the system.

\section{detection device}

The overall detection device is shown in Figure 1.The light source is composed of a light source, a condenser, a filter, and a small hole. The light is emitted from the hole position, reflected by plane mirror, collimated into parallel light by the large aperture collimator .It is the principle of collimator that a light source is imaged at the focal point of a parabolic mirror by mirror reflection, and the ray is projected onto a parabolic mirror by a flat mirror, then a parallel light with high accuracy is formed. In order to facilitate the detection and adjustment, all the detecting device is fixed on the air flotation optical platform. The large diameter optical system to be measured can be fixed on the adjustable bracket. The bracket can make front and rear adjustment and rotation adjustment. Parallel light focuses on actual focal plane, through the large aperture off-axis reflection system, then experimental personnel adjust the image analyzer to the focus by three dimensional image analyzer adjusting mechanism. Experimental personnel observe the location of the image points, until the image points on the computer software meet the requirements.MTF curve was obtained by software analysis by collecting the image. The whole system is composed of a light source generator and planar reflector and bearing, parabolic collimator, test platform, image analyzer and a bracket and computer and the software part. The overall system is based on the optical platform, so that it can adjust the position of each other more conveniently. The basic structure diagram, as shown in figure 1.

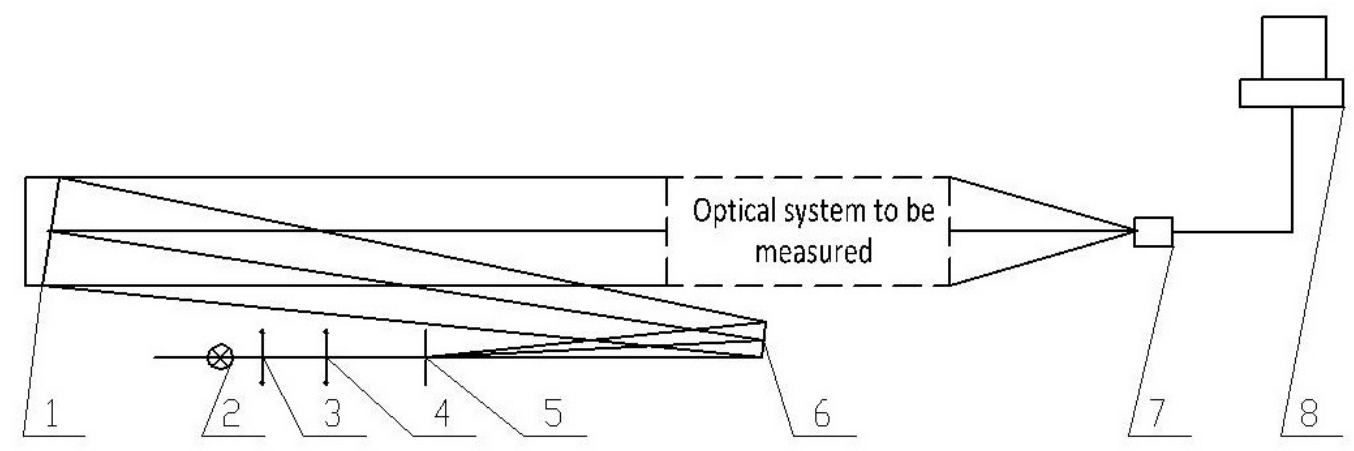

1-Large aperture parabolic mirror 2-Light source 3-Condenser 4-Filter 5-Target small hole 6-Planar mirror 7-Image analyzer and adjusting bracket 8-Computer and software processing part

Figure 1The transfer function of the detection principle 


\section{optical design and evaluation of detection system}

The detection system's design target requires that parallel optical aperture is $500 \mathrm{~mm}$, the focal length is controlled at $5000 \mathrm{~mm}$, the divergence angle is controlled at $0.02 \mathrm{mrad}$. The optical system of the detection system is a dual system of the off-axis. The main mirror is a parabolic mirror, the secondary mirror is a plat mirror. The light emitted from the light source is reflected by a mirror to a parabolic mirror, and turns into parallel light emitted into the mirror, and finally is imaged onto the CCD. The plane mirror is not light obscuration, Horizontal ray and planar mirror plane mirror bracket position left enough distance.

According to the detection and design requirements, selecting the plane mirror and the mirror of the axis of the mirror and the angle of the plane mirror, should be adjusted and optimized in the design process. The main parameters of the optical system are shown in Table 1.

Table 1Main parameters of off-axis parabolic mirror and plane mirror

\begin{tabular}{|c|c|c|c|c|c|}
\hline $\begin{array}{c}\text { Face serial } \\
\text { number }\end{array}$ & $\begin{array}{c}\text { Radius of } \\
\text { curvature[mm] }\end{array}$ & Thickness[mm] & Caliber[mm] & $\begin{array}{c}\text { Asphericcity } \\
\text { coefficient }\end{array}$ & $\begin{array}{c}\text { Off } \\
\text { axis[mm] }\end{array}$ \\
\hline Main mirror & -10000 & -4660 & 502 & -1 & -378.9 \\
\hline Planar mirror & & 340 & 62 & & 25.8 \\
\hline
\end{tabular}

The transfer function and diffraction energy distribution of the optimized system are shown in figure 2. Maximum disc of confusion of $6.656 \mu \mathrm{m}$, which is less than Airy disk $7.183 \mu \mathrm{m}$. The aberration correction is good, the light energy distribution is uniform, the optical system energy convergence is nice, the aberration is small, and picture element is good. The lowest ray of Parallel light is $80 \mathrm{~mm}$ from the plane mirror, that meet the requirements of mechanical and light obscuration allowance.

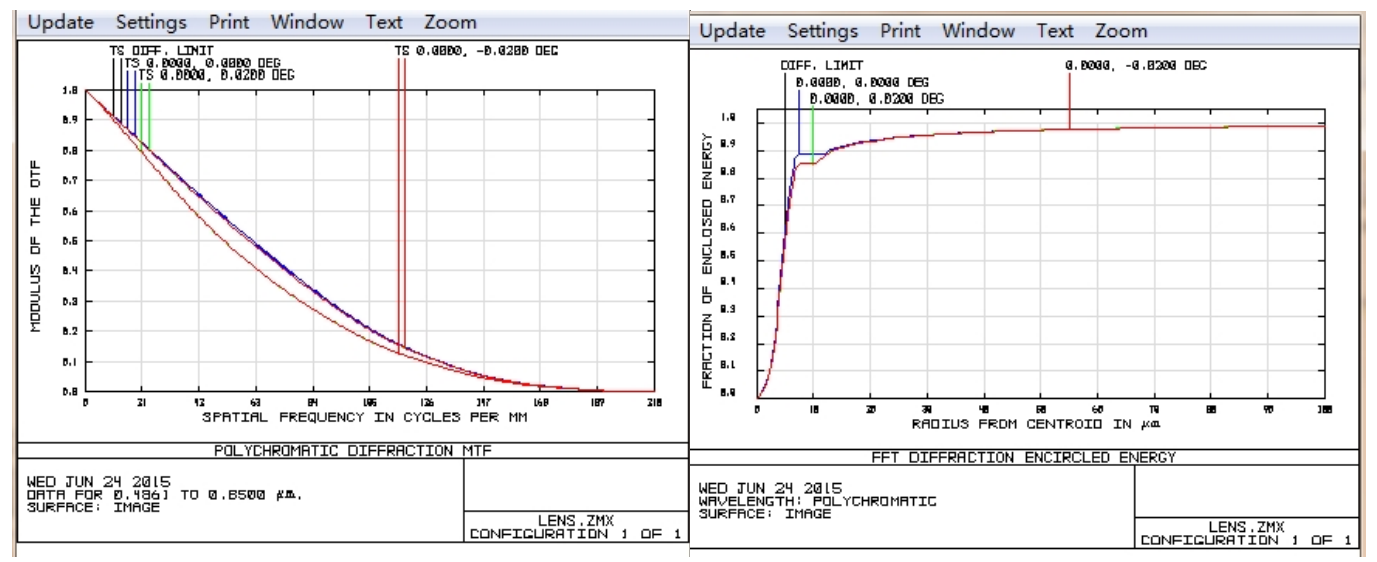

Fig. 2 system transfer function and system diffraction energy distribution

\section{System analysis and calculation}

The position of light source, condenser, filter and small pinholeare fixed, also these objects coupled with target pinhole are fixed on a flat mirror. Due to the diameter of the pinhole too small, when consider the angle and position of the plane mirror, see this pinhole as a point. The plane mirror is vertical to the air floating platform, and the angle between its normal line and the vertical direction is $\gamma$, it reflects the conical laser emitted from the light source, and shoots it onto the reflecting collimator. The parallelism of the rays is very important to the detection accuracy of transfer function, and the position error of the plane mirror is greatly affected the parallelism of the rays. 
The relative position of the small pinhole and the plane mirror can be adjusted according to the structural requirement, the relative position of the two is as follows: as seen in Fig.3, assuming that the focal point of the parabolic mirror is the original point; the coordinate of planar mirror center position is $(\mathrm{x}, \mathrm{y})$; The angle between normal line of plane mirror and a vertical is $\gamma$. By the theory of geometric optics, virtual image of pinhole at the focal point, That is the origin of Fig.3,The actual coordinates of the pinhole can be expressed as :

Horizontal coordinates:

$$
\mathrm{a}=\frac{2 \tan \gamma(x+y \tan \gamma)}{\sec ^{2} \gamma}
$$

Longitudinal coordinate:

$$
\mathrm{b}=\frac{2(x+y \tan \gamma)}{\sec ^{2} \gamma}
$$

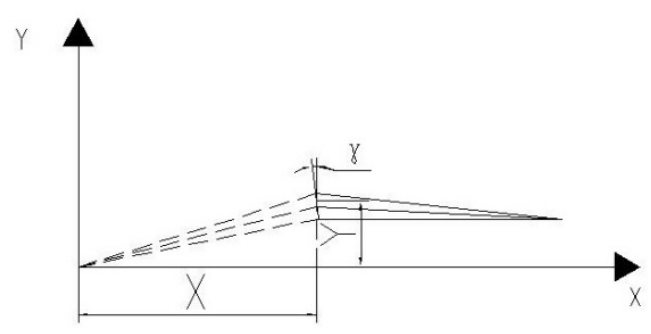

Fig. 3 Coordinate diagram of planar mirror and small pinhole position

To have a better optimization of the optical system, the following is the analysis of off axis value and the diameter of the pinhole influence to the divergence angle of the parallel light of the system. The analysis is based on geometrical optics principle and structure relationship.

Assuming that the focal point of the parabolic mirror is the original point; the horizontal axis is the $\mathrm{X}$, the vertical axis is the $\mathrm{Y}$, and the parabolic mirror is $\mathrm{f}$, represent the aperture diameter as the change of point S's coordinate. As is shown in Fig. 4.

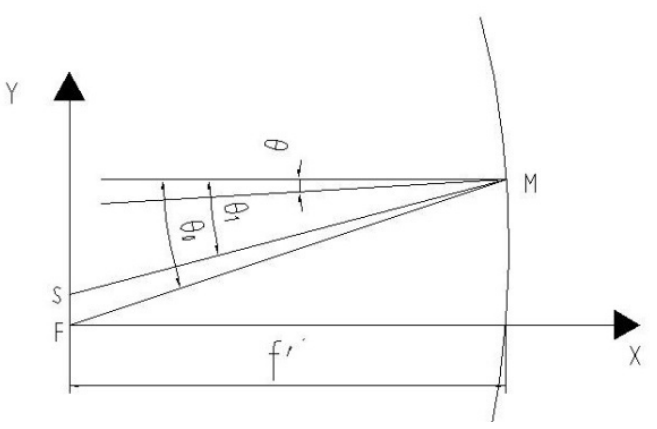

Fig.4 Coordinate diagram of exit light and reflected light

The equation of parabolic mirror center position is

$$
y^{2}=-4 f^{\prime}\left(x-f^{\prime}\right)
$$

The focal point is the original point, after imaged by planar mirror, the position of target pinhole's virtual image is $\mathrm{x}=0$. The light emitted from the focus position is folded by the paraboloid, 
the position of the exit light on the paraboloid is $\mathrm{M}\left(\mathrm{x}_{0}, \mathrm{y}_{0}\right)$. So, the equation of incident ray is

$$
y=\frac{4 f y_{0}}{4 f^{\prime 2}-y_{0}^{2}} x
$$

The light from the focal point can become a parallel light by a parabolic reflector, this means the ideal position of the light source is the focal point of a parabolic mirror. Meanwhile, the light from point $\mathrm{S}\left(\mathrm{x}_{1}, \mathrm{y}_{1}\right)$ folded by the paraboloid is now on point $\mathrm{M}\left(\mathrm{x}_{0}, \mathrm{y}_{0}\right)$. The slope of the incident light from $\mathrm{S}$ to $\mathrm{M}$ is:

$$
k_{1}=\frac{y_{0}-y_{1}}{x_{0}}=\frac{4 f^{\prime}\left(y_{0}-y_{1}\right)}{4 f^{\prime 2}-y_{0}^{2}}
$$

The light emitted from focal $\mathrm{F}(0,0)$ to $\mathrm{M}\left(\mathrm{x}_{0}, \mathrm{y}_{0}\right)$ is folded into horizontal light, the angle between the incident light and the horizontal light is $\theta_{0}$; after the point $\mathrm{S}\left(0, \mathrm{y}_{1}\right)$ on the source folded by $\mathrm{M}\left(\mathrm{x}_{0}, \mathrm{y}_{0}\right)$, he incident light and the horizontal light is $\theta_{1}$. The normal line of point $\mathrm{M}$ is fixed. According to the geometrical optics, the angle $\theta$ between emitting light and horizontal can be derived. $\theta_{0}$ can be obtained by slopek $_{0}, \mathrm{k}_{1}$, that is

$$
\theta=\theta_{0}-\theta_{1}=\arctan \mathrm{k}_{0}-\arctan \mathrm{k}_{1}
$$

The interval of $\theta, \theta_{0}, \theta_{1}$ is $\left(0, \frac{\pi}{2}\right)$;

The slope of the beam is derived by the sine formula, that is:

$$
k=\frac{k_{0}-k_{1}}{1+k_{0} k_{1}}=\frac{4 f y_{1}\left(4 f^{\prime 2}-y_{0}{ }^{2}\right)}{\left(4 f^{\prime 2}+y_{0}{ }^{2}\right)^{2}-1 f^{\prime 2} y_{0} y_{1}}
$$

the system requires the divergence angle is $0.02 \mathrm{mrad}$.Because value is very small, it can be approximated that $|\mathrm{k}|=\tan \theta \approx \theta$. According to the optical parameter on table 1, take focal length, off-axis quantity, divergence angle into formula(11), then the maximum value of y1 can be confirmed as $0.100431 \mathrm{~mm}$.

In this system, the diameter of the pinhole is $40 \mu \mathrm{m}$, which is Far less than $2 \mathrm{y} 1$. So the diameter of target pinhole can meet the requirements of the device.

Through the above analysis and calculation, only the divergence angle of the system is less than $0.02 \mathrm{mrad}$, the diameter of pinhole is below $200 \mu \mathrm{m}$, the design can meet the requirements. The diameter of the pinhole is $40 \mu \mathrm{m}$, which is satisfied.

\section{Design and selection of support and fine tuning mechanism}

According to the above analysis, Light source support adjusting mechanism need angle adjustment and position adjustment. fine tuning mechanism is applied to position adjustment to high obtain displacement positioning accuracy.

The light source platform is composed of a heavy load rotating table, a linear motion guide, a moving base and a stepping motor. the light source is fixed on moving base. Rotary table is a high precision electric rotary table with precision $0.0005^{\circ}$ and rotating range $\pm 90^{\circ}$.two-phase hybrid stepper motor is selected, which Connects with the drive ball screw by shaft coupling. Then, driven the light source part fixed on high precision linear motion guide to adjust before and after. The ball screw is used as the ball movement, so the starting torque is small, there will not be crawling 
phenomenon, which can ensure accurate micro feed. Micro feed rate can reach $0.01 \mathrm{~mm}$ that meets the system requirements. The basic structure picture is shown in Fig.5.

Large aperture reflecting collimator is consisted of a parabolic reflector with caliber $502 \mathrm{~mm}$, and its supporting structure. The aperture of the collimator is greater than the entrance pupil diameter of measured optical system. The reflect mirror is coating reflective film, the supporting structure comprises a base plate, vertical plate, stiffener, mirror seat, cover plate etc. The support structure is fixed on the optical platform, to ensure the overall structure and the vertical of optical platform, which facilitates the subsequent measurement and adjustment. The structure is shown in Fig.5.

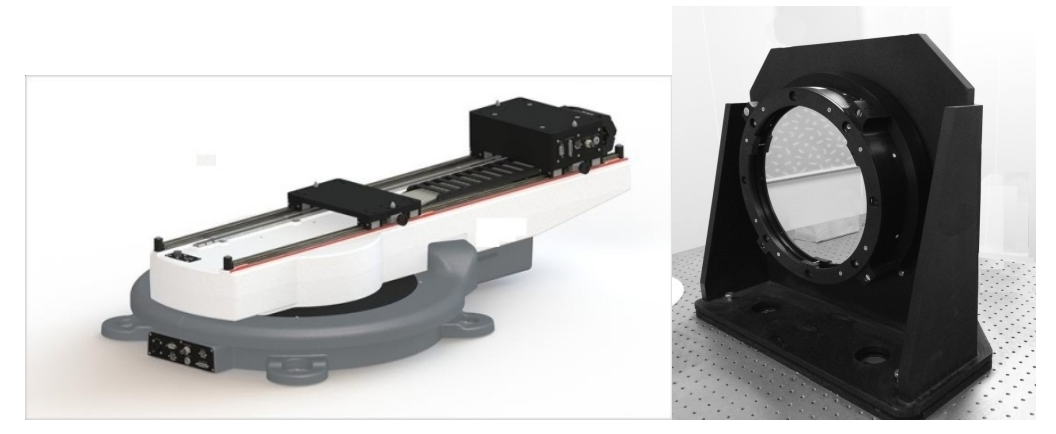

Fig. 5 light source fixed and fine adjustment mechanism \& mirror bracket structure diagram

\section{Conclusion}

In order to detect the large caliber optical system's transfer function, a transfer function detecting system with $500 \mathrm{~mm}$ caliber is designed. According to the technical requirements, basic parameters of each component can be confirmed, as well as position requirements for each component and specific structural parameters. The imaging quality of this system is good; the divergence angle can meet the requirements; it has a variety of fine tuning device; and easy to operate the measurement.

Besides, this detecting system can effectively control system aberration, and reduce the number of optical parts of the optical system. It also achieves large diameter, low obscuration ratio, compact optical system, and so on.

\section{Literature References}

[1] Paul R.Yoder, JR, Opto-Mechanical Systems Design, CRC Press. Beijing, 2006. in Chinese

[2] Yu Jian, Zhang Guoyu. Calibration of small field of view infrared probe with off-axis reflective collimator design [J]. Journal of Changchun University of Science and Technology, 1(2010)8-10. in Chinese

[3] Zhang Xingde, Li Ronggang, Wang Cheng. Research progress and technology of off-axis reflective optical system [J]. laser and infrared, 2(2013)128-131. in Chinese

[4] Gongquan Wang. CCD optical modulation transfer function and detection method [D]. Graduate University of Chinese Academy of Sciences, 2002.in Chinese 